\begin{tabular}{|c|c|c|}
\hline \multirow{3}{*}{$\begin{array}{r}\text { Case Reports in } \\
\text { Gastroenterology }\end{array}$} & \multirow{2}{*}{\multicolumn{2}{|c|}{ Case Rep Gastroenterol 2014;8:61-66 }} \\
\hline & & \\
\hline & $\begin{array}{l}\text { DOI: 10.1159/000354724 } \\
\text { Publisned online: February 13, } 2014\end{array}$ & $\begin{array}{l}\text { (c) } 2014 \text { S. Karger AG, Basel } \\
\text { 1662-0631/14/0081-0061 } \$ 39.50 / 0 \\
\text { www.karger.com/crg }\end{array}$ \\
\hline & \multicolumn{2}{|c|}{$\begin{array}{l}\text { This is an Open Access article licensed under the terms of the Creative Commons } \\
\text { Attribution-NonCommercial } 3.0 \text { Unported license (CC BY-NC) (www.karger.com/OA } \\
\text { license), applicable to the online version of the article only. Distribution permitted for non } \\
\text { commercial purposes only. }\end{array}$} \\
\hline
\end{tabular}
commercial purposes only.

\title{
Gastrointestinal and Extragastrointestinal Stromal Tumors: Report of Two Cases and Review of the Literature
}

\author{
Petros Antonopoulos $^{\mathrm{a}} \quad$ Polytimi Leonardou $^{\mathrm{a}}$ Nikolaos Barbagiannis $^{\mathrm{a}}$ \\ Konstantinos Alexiou ${ }^{b} \quad$ Maria Demonakou $^{c}$ Nikolaos Economou $^{b}$ \\ Departments of ${ }^{a}$ Computed Tomography and Magnetic Resonance Imaging,

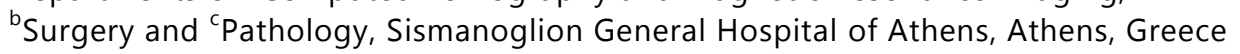

\section{Key Words}

Gastrointestinal stromal tumors · Extragastrointestinal stromal tumors · Computed tomography incidental findings · Stomach tumors · Hepatogastric ligament tumors

\begin{abstract}
We present two cases, one of a gastrointestinal stromal tumor (GIST) in the stomach and one of an extragastrointestinal stromal tumor (EGIST) in the hepatogastric ligament, which were discovered as incidental findings during computed tomography (CT) scans performed for other reasons. In both cases the diagnosis of the tumor was confirmed histologically and immunohistochemically. During the follow-up CT examinations these tumors proved to have a completely different natural course. The first case refers to an 82-year-old male patient with GIST of the stomach who refused to be operated and was followed by CT scans for a 4-year period. This patient did not show any significant changes in the morphology, size and density of the lesion. The second case refers to a 58-year-old female patient with EGIST of the hepatogastric ligament who presented with simultaneous liver metastases and remained healthy for 2 years after surgical resection, but developed local recurrence later. As a conclusion, both GISTs/EGISTs can be revealed as incidental findings in a CT scan performed for other purposes. Moreover, an untreated GIST located in the stomach can remain unchanged and without metastatic lesions for a long period of time, as in our case for a 4-year period. To our knowledge, this is the first report in the literature in whom a GIST was proved to remain almost unchanged for many years without any treatment, and we therefore attempt a further review of the current literature on stromal tumors.
\end{abstract}


Antonopoulos et al.: Gastrointestinal and Extragastrointestinal Stromal Tumors: Report of Two Cases and Review of the Literature

\section{Introduction}

Gastrointestinal stromal tumors (GISTs) are the majority of mesenchymal tumors of the gastrointestinal tract [1]. GISTs present in adults over 40 years (mean 55-60 years) and only exceptionally in children. The majority present in the stomach (60-70\%) or small intestine (25-35\%). The colon, rectum, appendix (together 5\%) and esophagus (2-3\%) are rare sites. Some GISTs are primary in the omentum, mesentery or retroperitoneum, but most GISTs in these sites are metastases from gastric or intestinal primaries [2-5]. Histologically they vary from cellular spindle cell tumors to epithelioid and pleomorphic ones, and morphology differs by site. By definition, GISTs are KIT (CD117)-positive. Positivity for nestin (90-100\%) and CD34 (70\%) are also characteristic but less specific features. Smooth muscle actins (20$30 \%$ ) and heavy caldesmon (80\%) are often expressed, whereas desmin is usually absent.

Stromal tumors arising outside the gastrointestinal tract are extremely rare and are known as extragastrointestinal stromal tumor (EGISTs); they account for less then $5 \%$ of all GISTs [2]. A high percentage of EGISTs represent a metastasis from a primary GIST. Histologically they resemble their gastrointestinal counterpart [2-4]. Approximately $80 \%$ of EGISTs are located in the omentum or mesentery, and the remainder develops in the retroperitoneum. They are reported to be located in different sites of the abdomen, however to our knowledge an EGIST located in the falciform ligament of the liver has been reported only once even in the recent literature [3, 4]. EGISTs located in the site of the hepatogastric ligament are often asymptomatic and are discovered incidentally during imaging scans performed for other reasons, i.e. during laparoscopic gastric bypass, at surgery and at autopsy.

The risk of malignant behavior of these tumors ranges from very low to high based on mitotic rate and size (tumors larger than $5 \mathrm{~cm}$ with more than 5 mitoses per $50 \mathrm{HPFs}$ are considered to be high-risk) and also on location (small bowel GISTs and EGISTS have a higher tendency for malignant behavior compared to those in the stomach) [5].

We hereby report two cases of stromal tumors of the abdomen which were discovered as incidental findings during computed tomography (CT) scan examinations. Both patients are aware of this report and signed an informed consent.

\section{Case Presentation}

Case 1

An 82-year-old male patient diagnosed with pulmonary carcinoma underwent a lung and abdominal CT examination for preoperative staging, which showed a round homogeneous soft tissue mass of $4.8 \times 5.5 \mathrm{~cm}$ in the stomach as an incidental finding (fig. 1). Gastroscopy confirmed the presence of the mass. An endoscopic biopsy was taken and histology showed positive immunohistochemical staining for CD117, a defining feature of GIST in the stomach. The patient was scheduled for lung cancer resection, however he refused any medical treatment of the gastric lesion. For the next 4 years he had CT follow-up examinations for both malignancies, which demonstrated that the gastric mass remained unchanged, without any significant differences in the morphologic characteristics.

Case 2

A 58-year-old female patient with a history of hypothyroidism, arterial hypertension and mitral regurgitation presented with left thoracic pain and fever up to $38.5^{\circ} \mathrm{C}$ during the last 3 days. Laboratory examination showed: WBC at the highest normal level $(10,100)$ with 
Antonopoulos et al.: Gastrointestinal and Extragastrointestinal Stromal Tumors: Report of Two Cases and Review of the Literature

elevated neutrophils count (81\%), increased erythrocyte sedimentation rate of 53 and elevated cancer antigen 125 of 8,300. A lung and abdominal CT scan was performed and showed an inhomogeneous mass sized $5.8 \mathrm{~cm}$ located in the hepatogastric ligament, in contact with the left liver lobe (fig. 2). The lesion demonstrated heterogeneity after intravenous contrast medium administration. Two small lesions of the liver compatible with hepatic metastases were also depicted. Moreover, the CT showed an extensive left pleural effusion and a myelolipoma of the left adrenal gland $3 \mathrm{~cm}$ in size. The patient underwent endoscopy, bronchoscopy and video-assisted thoracic surgery, which did not reveal any pathological findings. Fine needle aspiration and biopsy of the mass proved immunohistochemical features of a CD117-positive mesenchymal tumor of the hepatogastric ligament without connection to the gastrointestinal tract, compatible with an extragastrointestinal tumor. Because of the presence of hepatic metastases, conservative therapy with Gleevec was initially decided.

\section{Discussion}

GISTs include most tumors previously designated as leiomyoma, cellular leiomyoma, leiomyoblastoma and leiomyosarcoma [1]. GISTs arise from the interstitial cells of Cajal and are the most common gastrointestinal mesenchymal tumors, ranging from 0.1 to $3 \%$ of all gastrointestinal tumors, more frequently located in the stomach [5]. The annual incidence of GISTs increased from 2.1 per million inhabitants in 1995 to 12.7 per million inhabitants in 2003 and the increased incidence during this period is related to the increased understanding of GIST pathobiology and the routine availability of the diagnostic immunohistochemical antibody directed against the CD117 antigen [6]. In Greece, about 250 GISTs are diagnosed annually. GISTs occur predominantly in middle-aged and older people, with an average age of $50-60$ years $[5,7]$.

GISTs have an uncertain clinical behavior, ranging from benign to malignant, and there is no accepted staging system. Initial size, age of the patient, anatomic location, mitotic activity, histologic subtype and presence of intratumoral necrosis were suggested as criteria for the prognosis, affecting overall survival [7]. Although presence of an ulcer, mesenteric fat infiltration, direct organ invasion and metastasis were more frequently observed in tumors with a high mitotic rate, small bowel GISTs have a high tendency for malignant behavior compared with those in the stomach. Tumors with a size larger than $5 \mathrm{~cm}$ have an especially poor prognosis, with decreased survival and increased local and/or distant recurrence. In a large study by Cao et al. [7], patients with GIST had tumors which ranged in size between 0.5 and $30 \mathrm{~cm}$ with a mean size of $7.02 \mathrm{~cm}$.

In our case, the patient refused to undergo surgery or receive any other treatment, and therefore this may be the first time that it was possible to observe the progress and development of a GIST over years, proving that there may be cases of GIST with very slow progression when left untreated.

CT is the imaging modality of choice for the diagnosis of a GIST, which may appear on CT as an exophytic mass, well or irregularly defined and enhancing either homogeneously or inhomogeneously after intravenous contrast medium administration. Less common findings include central necrosis of the tumor, dilation of the bowel, ascites and omental caking [8]. Fluorine-18-fluorodeoxyglucose positron emission tomography is sometimes useful for the evaluation of GIST outcomes and is indicated when CT findings are inconsistent with the clinical presentation or are inconclusive [8]. 
Antonopoulos et al.: Gastrointestinal and Extragastrointestinal Stromal Tumors: Report of Two Cases and Review of the Literature

Hepatic spread is the most common metastasis of GISTs. Following STI-571 treatment, the liver metastases can appear on CT as cystic hepatic lesions. The hepatic metastases can either decrease in size after treatment or rarely remain unchanged in size [9].

Because of the fact that GISTs often occur with nonspecific symptoms, the diagnosis of these tumors is frequently delayed. The clinical manifestations of GIST depend on the size and anatomic location of the tumor and include gastrointestinal bleeding, hematemesis, melena, hematochezia, a palpable mass, abdominal pain, vomiting, weight loss, signs and symptoms of anemia, abdominal distention and intestinal obstruction [10]. The CT findings, such as the size and localization of tumor and the presence of metastases, play a crucial role in the choice of the treatment. Oncologic complete surgical resection is the gold standard treatment for primary nonmetastatic GISTs [11].

The treatment with administration of the KIT-selective tyrosine kinase inhibitor imatinib mesylate (STI-571), commercially known as Gleevec/Glivec (Novartis Pharma, Basel, Switzerland), has proved to be effective in the treatment of unresectable and metastatic tumors. Blanke et al. [12] reported that nearly $50 \%$ of patients with an advanced GIST who were treated with imatinib mesylate survived for more than 5 years. Local recurrence may occur after surgical resection of a GIST. Tumor size, cell proliferation index and location of the tumor can be used to predict the risk of recurrence in GIST patients. Recurrence of a GIST is usual, and a report by $\mathrm{Ng}$ et al. [13] found only $10 \%$ of patients to be free of disease after a long-term follow-up. Another study by Dematteo et al. [5] showed that 30\% of the patients with GIST had recurrent disease within 2 years and approximately half of the patients who have undergone complete resection will survive for more than 5 years. The high rate of local and distant recurrence underscores the need for adjuvant therapy.

EGISTs are very rare tumors, and a large study by Agaimy and Wunsh [14] reports that they account for $1.5 \%$ of total GISTs. These tumors may appear in different sites of the abdomen such as in the pancreas, spleen, retroperitoneum, mesocolon, mesentery of the small intestine, mediastinum and in the pelvis as vulvovaginal/rectovaginal septal masses [15]. EGISTs usually behave more aggressively. Factors that indicate poor prognosis include tumor size greater than $5 \mathrm{~cm}$, mitotic rate greater than 1-5/10 HPFs, presence of tumor necrosis or metastasis and most recently the c-kit mutation. The majority of EGISTs (14 cases) that were re-classified by Agaimy and Wunsh [14] were found to be either GISTs with extensive extramural growth, resulting in loss of contact with the external muscle coat of the gut $(8 / 14)$, or a metastasis from an inoperable GIST $(2 / 14)$ or from a previously resected deceptively benign tumor $(1 / 14)$.

As a conclusion, GISTs and EGISTs may be revealed as incidental findings in a CT scan performed for other reasons. GIST can remain unchanged, with no presence of metastatic lesions, if not receiving any treatment and followed up for a 4-year period. EGIST located in the hepatogastric ligament may present with metastases to the liver at the time of discovery.

\section{References}

1 Miettinen M, Lasota J: Gastrointestinal stromal tumors - definition, clinical, histological, immunohistochemical, and molecular genetic features and differential diagnosis. Virchows Arch 2001;438:1-12.

2 Devanand B, Vadiraj P: The cytology of the benign extra-gastrointestinal stromal tumour in the pouch of Douglas: a case report. J Clin Diagn Res 2011;5:1659-1661.

-3 Abedalthagafi M: Gastrointestinal stromal tumour originating from the hepatic falciform ligament. BM] Case Rep DOI: 10.1136/bcr-03-2012-6136.

-4 Munoz M, Ramirez PT, Echeverri C, Alvarez LG, Palomino MA, Pareja LR: Gastrointestinal stromal tumors as an incidental finding in patients with a presumptive diagnosis of ovarian cancer. J Gynecol Oncol 2012;23:48-52. 


\begin{tabular}{l|l}
\hline \multicolumn{2}{l}{ Case Rep Gastroenterol 2014;8:61-66 } \\
\hline DOI: 10.1159/000354724 & $\begin{array}{l}\text { @ 2014 S. Karger AG, Basel } \\
\text { www.karger.com/crg }\end{array}$ \\
\hline
\end{tabular}

Antonopoulos et al.: Gastrointestinal and Extragastrointestinal Stromal Tumors: Report of Two Cases and Review of the Literature

5 Dematteo RP, Lewis JJ, Leung D, Mudan SS, Woodruff JM, Brennan MF: Two hundred gastrointestinal tumors: recurrence patterns and prognostic factors for survival. Ann Surg 2000;231:51-58.

-6 Goettsch WG, Bos SD, Breekvedt-Postma N, Casparie M, Herings RM, Hogendoorn PC: Incidence of gastrointestinal stromal tumours is underestimated: results of a nation-wide study. Eur J Cancer 2005;41:2868-2872.

-7 Cao H, Zhang Y, Wang M, Shen DP, Sheng ZY, Ni XZ, Wu ZY, Liu Q, Shen YY, Song YY: Prognostic analysis of patients with gastrointestinal stromal tumors: a single unit experience with surgical treatment of primary disease. Chin Med J (Engl) 2010;123:131-136.

8 Hong X, Choi H, Loyer EM, Benjamin RS, Trent JC, Charnsangavej C: Gastrointestinal stromal tumor: role of CT in diagnosis and in response evaluation and surveillance after treatment with imatinib. Radiographics 2006;26:481-495.

-9 Bechtold RE, Chen MY, Stanton CA, Savage PD, Levine EA: Cystic changes in hepatic and peritoneal metastases from gastrointestinal stromal tumors treated with Gleevec. Abdom Imaging 2003;28:808-814.

-10 Levy AD, Remotti HE, Thompson WM, Sobin LH, Miettinen M: Gastrointestinal stromal tumors: radiologic features with pathologic correlation. Radiographics 2003;23:283-304, 456.

-11 Wu PC, Langerman A, Ryan CW, Hart J, Swiger S, Posner MC: Surgical treatment of gastrointestinal stromal tumors in the imatinib (STI-571) era. Surgery 2003;134:656-665.

-12 Blanke CD, Demetri GD, von Mehren M, Heinrich MC, Eisenberg B, Fletcher JA, Corless CL, Fletcher CD, Roberts PJ, Heinz D, Wehre E, Nikolova Z, Joensuu H: Long-term results from a randomized phase II trial of standard- versus higher-dose imatinib mesylate for patients with unresectable or metastatic gastrointestinal stromal tumors expressing KIT. J Clin Oncol 2008;26:620-625.

13 Ng EH, Pollock RE, Romsdahl MM: Prognostic implications of patterns of failure for gastrointestinal leiomyosarcomas. Cancer 1992;69:1334-1341.

14 Agaimy A, Wunsh PH: Gastrointestinal stromal tumours: a regular origin in the muscularis propria, but an extremely diverse gross presentation. A review of 200 cases to critically re-evaluate the concept of so-called extra-gastrointestinal stromal tumours. Langenbecks Arch Surg 2006;39:322-329.

15 Barros A, Linhares E, Valadão M, Gonçalves R, Vilhena B, Gil C, Ramos C: Extragastrointestinal stromal tumors (EGIST): a series of case reports. Hepatogastroenterology 2011;58:865-868.

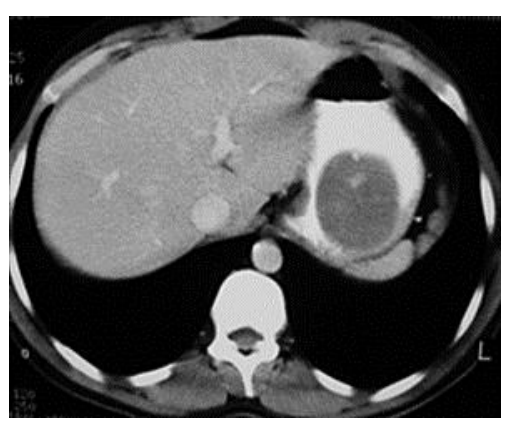

Fig. 1. CT of the abdomen after intravenous contrast medium administration demonstrated a homogeneous, round, soft tissue density mass in the stomach, with intratumoral solitary focal calcification and very smooth margins, $4.8 \times 5.5 \mathrm{~cm}$ in size, which presented homogeneous enhancement. 


\begin{tabular}{l|l}
\hline Case Rep Gastroenterol 2014;8:61-66 \\
\hline DOI: 10.1159/000354724 & $\begin{array}{l}\text { @ 2014 S. Karger AG, Basel } \\
\text { www.karger.com/crg }\end{array}$ \\
\hline
\end{tabular}

Antonopoulos et al.: Gastrointestinal and Extragastrointestinal Stromal Tumors: Report of Two Cases and Review of the Literature

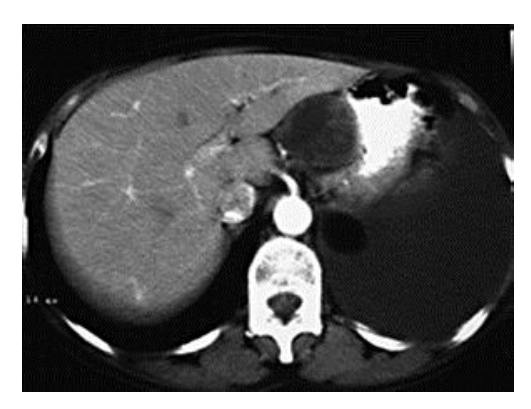

Fig. 2. CT of the abdomen after intravenous contrast medium administration showed an inhomogeneous mass with heterogeneous enhancement, located between the stomach and the liver, in the area of the hepatogastric ligament. 